\title{
Hot Topics in Epilepsy-Highlights of the American Epilepsy Society Annual Meeting 2018
}

\author{
Neishay Ayub, ${ }^{1}$ Daniel Goldenholz, ${ }^{2}$ Shimrit Uliel-Sibony, ${ }^{3}$ Martha Sajatovic, ${ }^{4}$ Hillary M Thomas, ${ }^{5}$ Susan Arnold, ${ }^{6}$ Paula E Voinescu, ${ }^{7}$ Zhibin Chen ${ }^{8}$ \\ 1. Department of Neurology, Massachusetts General Hospital, Boston, MA, USA; 2. Department of Neurology, Beth Israel Deaconess Medical Center, Boston, MA, USA; \\ 3. Pediatric Epilepsy Service, Dana-Dwek Children's Hospital, Tel Aviv Sourasky Medical Center, Tel Aviv-Yafo, Israel; 4. Psychiatry-Adult Psychiatry, Neurological and \\ Behavioral Outcomes Center, Case Western Reserve University School of Medicine, Cleveland, OH, USA; 5. University of Texas Southwestern Medical Center, Dallas, TX, USA, \\ 6. Department of Neurology and Neurotherapeutics, University of Texas Southwestern Medical Center, Dallas, TX, USA; 7. Department of Neurology, Brigham and Women's \\ Hospital, Harvard University, Boston, MA, USA; 8. Department of Neuroscience, University of Melbourne, VIC, Australia.
}

\section{Keywords}

Epilepsy, cannabidiol, depression, seizure, pregnancy, sudden unexplained death

\section{Disclosure: Daniel Goldenholz discloses funding in part from NIH T32NS048005. Neishay Ayub, Shimrit Uliel-Sibony, Martha Sajatovic, Hillary Thomas, Susan Arnold, Paula E Voinescu, and Zhibin Chen have nothing to declare in relation to this article. \\ Review Process: This is a series of expert interviews and as such, has not undergone the journal's standard peer review process. \\ Acknowledgements: Special thanks to Caitlin Blum on behalf of the American Epilepsy Society. \\ Authorship: All named authors meet the criteria of the International Committee of Medical Journal Editors for authorship for this manuscript, take responsibility for the integrity of the work as a whole and have given final approval for the version to be published. \\ Received: February 6, 2019 \\ Accepted: March 12, 2019 \\ Citation: US Neurology. 2019;15(1):22-5 \\ Corresponding Author: Eileen Murray, American Epilepsy Society, 135 South LaSalle Street, Suite 2850, Chicago, IL 60603, USA. E: emurray@aesnet.org}

Support: No funding was received in the publication of this article.
The American Epilepsy Society Annual Meeting is the largest gathering on epilepsy in the world. The 2018 meeting, which was held in New Orleans, Louisiana, from November 30-December 4, 2018, featured symposia, workshops, and lectures aimed at healthcare practitioners involved in all aspects of the care of patients with epilepsy. In a number of expert interviews conducted at the meeting, authors of key presentations shared their expertise and provided insights into some of the hottest topics in the field.

\section{Cannabidiol benefit may decline}

\section{Scimrit Uliel-Sibony}

Lead author of study on cannabidiol-enriched oil effectiveness in children and adults with treatmentresistant epilepsy. ${ }^{1}$

Q. In which children with epilepsy is cannabidiol (CBD) indicated?

Any child or adult who has failed adequate trials of at least two tolerated, appropriately chosen, antiseizure medications, which were given adequately, is considered to have refractory epilepsy. This typically affects $20-30 \%$ of patients. ${ }^{2}$ An open-label trial showed that CBD reduced the frequency of seizures over 12 weeks in children and young adults with refractory epilepsy. ${ }^{3}$

Q. Could you tell us a little about your recent study on the effectiveness of CBD in epilepsy over time and its findings?

In our study, 92 children and young adults with treatment-resistant seizures took CBD for an average of 19.8 months. We found that in about $25 \%$ of our patients, we had to increase the CBD dose to maintain the initial efficacy; however, dosage increment achieved the previous response in only four patients (19\%) and a satisfying but less than prior response in 10 patients (47\%). ${ }^{1}$

Q. Were any factors identified that could predict which patients were likely to develop resistance?

Unfortunately, no correlation between tolerance development and age at treatment onset, etiology, clobazam treatment, or treatment duration was found, but patients with shorter epilepsy duration showed a higher tendency to develop tolerance. ${ }^{1}$

Q. What are the treatment options for patients who develop resistance? Refractory epilepsy is challenging to treat. Benefits have been reported with ketogenic diet therapies, ${ }^{4}$ vagal nerve stimulation, ${ }^{5}$ and steroids. ${ }^{6}$

Q. What is the long-term implication of these findings and what future studies are planned?

since tolerance is a well-known phenomenon in epilepsy treatment, it means that we will have to continue to look for better, long-term solutions for our patients. We are planning to investigate the different components of the cannabis extract and try to isolate the exact active element in the treatment of different refractory epilepsy syndromes. $\square$ 


\section{Epilepsy increases depression risk}

\author{
Martha Sajatovic, Hilllary M Thomas, Susan Arnold
}

Lead author and co-authors of the study of teens and adults with epilepsy and increased risk of depression.?

\section{Q. What were the findings of the study of depression risk in teenagers with epilepsy, and how does this compare to the risk in the general population?}

The study assessed 394 teenagers (aged 15-18) with epilepsy and found that $13 \%$ of teenagers reported serious depression symptoms, and an additional $22 \%$ of teens with epilepsy scored high enough results to suggest a risk for depression. ${ }^{7}$ This compares with a risk of $7.6 \%$ among people over age 12 in the general population. ${ }^{8}$

\section{Q. What were the findings of the SMART study, a} separate study of depression in adults with epilepsy? The SMART study is a baseline analysis from a prospective randomized controlled study that measured depression severity using the Patient Health Questionnaire-9 (PHQ-9). This is a widely used instrument that has been used to both screen for and monitor depressive severity in populations with chronic health conditions, including epilepsy and demographic and clinical variables. ${ }^{9}$ Approximately half had clinically significant depression as measured by established thresholds on the PHQ-9.10

\section{Q. How does the incidence of depression affect the frequency of seizures in patients with epilepsy?}

The presence of seizures in the past 30 days correlated with increased depression severity such that a higher number of seizures was correlated with worse depression severity. ${ }^{10}$ This is in line with other studies that suggest that having more seizures is associated with worse depression. ${ }^{11}$ While our study could not determine a definitive causal link between those factors, one might also speculate that these two things feed into each other. If a person is depressed, they may be less likely to have the energy and motivation to address the multiple things that people with epilepsy have to do for optimal health. For example, taking antiepileptic drugs (AEDS) on time, taking the effort to ensure a healthy diet, getting the right amount of sleep, keeping doctor's appointments, and so on. You could see how that could make seizure control even worse and things just spiral down from there.

\section{Q. On the basis of these findings, how should clinicians monitor depression in their patients with epilepsy?}

Our findings potentially inform the clinical management of people with epilepsy and emphasize the importance of screening tools for depression and other psychiatric conditions in standard clinical settings. It is also important to mention that screening should not be a one-time event, but rather something that should be done at regular intervals in clinical settings. Standardized self-rated depression measures, like the PHQ-9 used in our study, are quick and practical. ${ }^{12}$

\section{Q. What strategies can be used to manage depression or other mental health issues in patients with epilepsy?}

It is important to remember that self-report tools like the PHQ-9 are screening instruments and that a more detailed clinical evaluation is warranted in patients who score in the moderate or higher range of depression severity. Treatments for depression can include a variety of medications as well as behavioral approaches like cognitive behavioral therapy. ${ }^{13}$ Our analysis suggests that higher levels of depressive symptom severity may occur in the context of other psychiatric conditions such as bipolar disorder, post-traumatic stress disorder, or obsessive-compulsive disorder in people with epilepsy. ${ }^{14}$ Specialist referral may be indicated in these instances, since some commonly used treatments, such as the use of antidepressant monotherapy, can worsen the clinical course of bipolar disorder. ${ }^{14}$

We have also recently been notified that a scientific manuscript summarizing our findings has been accepted for publication in the Journal of Nervous and Mental Disorders. I do not have a publication date but expect it will be 2019.

\section{Some pregnant women are at a high risk for seizures}

\section{Paula E Voinescu}

Lead author of the study on whether women with frontal lobe epilepsy are most likely to have more seizures while pregnant. ${ }^{15}$

\section{Q. What are the health risks associated with seizures in pregnancy?}

First, the unequivocal risk for trauma is affecting not only the mother, but the fetus as well, with an added risk for premature labor, infection, and fetal death. ${ }^{16}$ Generalized convulsions can cause maternal hypoxia and acidosis, also affecting the fetus and leading to possible fetal heart rate decelerations and miscarriages. ${ }^{17}$ In addition to the physical risks, there is one study suggesting that numerous generalized convulsions may impair neurodevelopment. ${ }^{18}$

\section{Q. What is the impact of pregnancy on the metabolism of AEDs?}

Pregnancy changes drug disposition through multiple physiological changes: alterations in drug absorption, increased volume of distribution, hepatic enzymatic induction via increased female sex steroid hormones, and increased renal blood flow and glomerular filtration rate. We showed that the time course and magnitude of the clearance changes is different for different AEDS and highlighted the importance of therapeutic drug monitoring in previous studies. ${ }^{19}$

\section{Q. Could you tell us a little about your study on \\ pregnant women with epilepsy, and its findings?}

This study is an analysis of prospectively collected clinical data on 114 pregnancies in 99 women with epilepsy followed in the Epilepsy-Obstetrics Clinics at Brigham and Women's Hospital between 2013-18. ${ }^{15}$ Seizure frequency worsening during pregnancy or postpartum was compared with preconception seizure frequency. Women with focal epilepsy have higher rates of seizure worsening during pregnancy compared with women with generalized epilepsy, and women with frontal lobe epilepsy have especially elevated rates of seizure worsening during pregnancy and postpartum. We ensured that in all women with seizure worsening, therapeutic drug monitoring was adequate. 
Q. Which women were at greatest risk of seizures and what is the reason for this?

Within the focal epilepsies, women with frontal lobe epilepsy have eighttimes the odds of increased seizure frequency during pregnancy compared with all other focal epilepsy. Polytherapy is also an indicator for a higher risk of seizure worsening, but individual monotherapies are not. When we refined our analysis to only women with focal epilepsy, we found that the relationship between having frontal focal epilepsy and increased seizure frequency in pregnancy persisted $(p=0.0105)$ even when polytherapy was added to the model..$^{15}$

\section{Epilepsy treatment gap exists in wealthy countries}

\section{Zhibin Chen}

Lead author of the study on the existence of untreated epilepsy in wealthy countries. ${ }^{20}$

\section{Q. What are the known benefits of prescribing an AED} at the time of diagnosis of epilepsy?

commencing an AED at the time of diagnosis will reduce the risk of further seizures that can cause injuries from impaired awareness or even be life-threatening, as uncontrolled seizure is a known risk factor for sudden unexpected death in epilepsy (SUDEP). ${ }^{21,22}$ Through avoiding further seizures, there will also be psychosocial benefits, e.g., returning to driving after a certain seizure-free period, minimizing impact on work performance, and improving quality of life. These benefits would need to be balanced against potential risks from medication side effects in decision-making for the individual patients.

\section{Q. According to your study, what proportion of patients are not receiving an AED prescription when diagnosed with epilepsy?}

Of the 610 patients newly diagnosed with epilepsy, 189 (31.0\%) were not prescribed AEDS at diagnosis. This proportion comprised 100 patients (16.4\%) who were not offered to start treatment by their neurologists, and 89 (14.6\%) who declined treatment despite recommendation. Among the 100 patients who were not recommended therapy at the time of diagnosis, the most common reasons were that despite a diagnosis of epilepsy, only a single seizure had occurred (30.0\%), the neurologist opted to await further results (29.0\%), and the presence of avoidable lifestyle/ external factors (22.0\%). Among the 89 patients who declined therapy at the time of diagnosis, the most common reasons were that the patients were unconvinced of the necessity for treatment (48\%), unconvinced of the diagnosis (17.0\%), and preferred to avoid lifestyle/external factors (13.0\%). ${ }^{20}$

\section{Q. Which patients were most likely to be prescribed} an AED?

Patients who had epileptogenic neuroimaging finding, more seizures, were older, and, perhaps counterintuitively, lived in areas with lower socio-economic status were more likely to commence an AED. Interestingly, epileptiform electroencephalogram (EEG) findings appeared to play a lesser role than epileptogenic neuroimaging abnormalities in the treatment commencement decision. The reason for this is unclear but might reflect the inherent differences in "weighting" ascribed by neurologists to different investigations in the treatment decision process. Socio-economic status was higher among patients not commencing treatment at diagnosis. It is possible that people with higher socio-economic status are more able to adjust their occupations to mitigate the potential harm from untreated recurrent seizures. ${ }^{22}$

\section{Q. What can be done to ensure more patients are prescribed AEDs?}

Communication is the key. Epilepsy treatment decision-making is a very complex process that requires neurologists to consolidate information from multiple sources, including seizure activity, diagnostic results, medical history, and the patient's lifestyle. When patients have doubts about the need for treatment, it is important for neurologists to clarify any misunderstandings. $\square$

\section{Risk of sudden unexpected death in epilepsy can change}

\section{Neishay Ayub and Daniel Goldenholz}

Lead author and co-author of the study on whether the risk of SUDEP may decrease over time. ${ }^{23}$

\section{Q. What are the known risk factors for SUDEP?}

A number of factors have been identified; however, there is still disagreement in the literature about some of them. A recent consensus statement published in 2017 by Harden et al. emphasizes these risk factors: ${ }^{24}$

- presence of generalized tonic clonic seizures (GTCS)

- yearly frequency of GTCS

- absence of seizure freedom

- not adding a new medicine when seizures are ongoing

- nocturnal supervision (this reduces risk)

- nocturnal listening device (this reduces risk).

Q. Could you tell us a little about the design of your recent study on SUDEP?

Data from one of the world's largest patient-reported seizure diary databases (SeizureTracker.com ${ }^{\mathrm{TM}}$ ) was analyzed. SUDEP risk levels were grouped as low (zero generalized seizures in a year), medium (1 or 2 generalized seizures in a year), and high ( $\geq 3$ generalized seizures in a year). We wanted to find out what percentage of patients remain in the same category over time. ${ }^{23}$

Q. According to the findings of your study, how does the risk of SUDEP change over time in different risk groups?

After monitoring for 3 years, low-risk patients tended to stay low risk (only $22 \%$ moved to a higher risk category), while high-risk patients tend to switch out of that risk category over time: $59 \%$ of patients in the high-risk group left their initial category.

Q. What are the clinical implications of these findings? Patients who do not tend to have GTCS are likely to have lower risk for SUDEP in the immediate year, but also in subsequent years. Patients with medium-high risk for SUDEP may change their risk category over time, so yearly assessment may be reasonable.

\section{Q. What future studies are planned?}

Future studies will investigate more comprehensive SUDEP risk assessments in this and other databases to better understand the longitudinal risk of SUDEP. $\square$ 
1. Uliel-Sibony S, Hausman-Kedem M, Kramer U. Cannabidiol tolerance in children and adults with treatment-resistant epilepsy. Presented at: The 72nd Annual Meeting of the American Epilepsy Society, New Orleans, Louisiana, US. November 30 - December 4 2018. Abstr 2.233.

2. Kwan $P$, Arzimanoglou $A$, Berg AT, et al. Definition of drug resistant epilepsy: consensus proposal by the ad hoc task force of the ILAE Commission on Therapeutic Strategies. Epilepsia. 2010;51:1069-77.

3. Devinsky O, Marsh E, Friedman D, et al. Cannabidiol in patients with treatment-resistant epilepsy: an open-label interventional trial. Lancet Neurol. 2016;15:270-8.

4. Falco-Walter JJ, Roehl K, Ouyang B, et al. Do certain subpopulations of adults with drug-resistant epilepsy respond better to modified ketogenic diet treatments? Evaluation based on prior resective surgery, type of epilepsy, imaging abnormalities, and vagal nerve stimulation. Epilepsy Behav. 2019; [Epub ahead of print] doi: 10.1016/j.yebeh.2019.01.010.

5. Arcand J, Waterhouse K, Hernandez-Ronquillo L, et al. Efficacy of vagal nerve stimulation for drug-resistant epilepsy: is it the stimulation or medication? Can J Neurol Sci. 2017:44:532-7.

6. Verhelst H, Boon P, Buyse G, et al. Steroids in intractable childhood epilepsy: Clinical experience and review of the literature seizure. 2005;14:412-21.

7. Thomas H, Sajatovic M, Arnold S. Depression screening in pediatric epilepsy. Presented at: The 72nd Annual Meeting of the American Epilepsy Society, New Orleans, Louisiana, US. November 30-December 4, 2018. Abstr 1.388 .
8. Centers for Disease Control and Prevention. Depression, 2016 Available at: www.cdc.gov/nchs/fastats/depression.htm (Accessed March 5, 2019)

9. Sajatovic M, Colon-Zimmermann K, Kahriman M, et al. A 6-month prospective randomized controlled trial of remotely delivered group format epilepsy self-management versus waitlist contro for high-risk people with epilepsy. Epilepsia. 2018;59:1684-95.

10. Kroenke K, Spitzer RL, Williams JBW. The PHQ-9: validity of a brief depression severity measure. J Gen Intern Med. 2001;16:606-613.

11. Kanner AM. Depression and epilepsy: A bidirectional relation? Epilepsia. 2011;52 Suppl.1:21-7.

12. Jamal-Omidi $S$, Collins $C$, Fulchiero $E$, et al. Assessing depression severity with a self-rated vs. rater-administered instrument in patients with epilepsy. Epilepsy Behav. 2018:85:52-7.

13. DeRubeis RJ, Siegle GJ, Hollon SD. Cognitive therapy versus medication for depression: Treatment outcomes and neural mechanisms. Nat Rev Neurosci. 2008;9:788-96.

14. Patel R, Reiss P, Shetty H, et al. Do antidepressants increase the risk of mania and bipolar disorder in people with depression? A retrospective electronic case register cohort study. BMJ Open. 2015;5:e008341.

15. Voinescu PE, Ehlert EN, Allien S, et al. Variations in seizure frequency during pregnancy and postpartum by epilepsy type. Presented at: The 72 nd Annual Meeting of the American Epilepsy Society, New Orleans, LA, USA. November 30-December 4, 2018. Abstr 3.236.

16. Sveberg $L$, Svalheim $S$, Tauboll E. The impact of seizures on pregnancy and delivery. Seizure. 2015;28:35-8.

17. Teramo $\mathrm{K}$, Hiilesmaa $\mathrm{V}$, Bardy $\mathrm{A}$, et al. Fetal heart rate during a maternal grand mal epileptic seizure. J Perinat Med. 1979;7:3-6 18. Lima DC, Vale TG, Arganaraz GA, et al. Behavioral evaluation of adult rats exposed in utero to maternal epileptic seizures. Epilepsy Behav. 2010;18:45-9.

19. Feghali $\mathrm{M}$, Venkataramanan $\mathrm{R}$, Caritis S. Pharmacokinetics of drugs in pregnancy. Semin Perinatol. 2015;39:512-9.

20. Chen $Z$. Untreated epilepsy exists even in wealthy countries, research shows. Presented at: The 72nd Annual Meeting of the American Epilepsy Society, New Orleans, LA, USA. November 30-December 4, 2018. Available at: www.aesnet.org/about_aes/ press_releases/untreated-epilepsy-wealthy-countries (accessed March 22, 2019)

21. Sperling MR. Sudden unexplained death in epilepsy. Epilepsy Curr. 2001;1:21-3.

22. Goldenberg MM. Overview of drugs used for epilepsy and seizures: Etiology, diagnosis, and treatment. PT. 2010;35:392-415.

23. Ayub N, Chiang S, Moss R, et al. Longitudinal SUDEP Risk Stratification with Electronic Diaries. Presented at: The 72nd Annual Meeting of the American Epilepsy Society, New Orleans, Louisiana, US. November 30 - December 4, 2018. Abstr 2.158.

24. Harden C, Tomson T, Gloss D, et al. Practice guideline summary: Sudden unexpected death in epilepsy incidence rates and risk factors: Report of the Guideline Development, Dissemination, and Implementation Subcommittee of the American Academy of Neurology and the American Epilepsy Society. Neurology. 2017;88:1674-80 